\title{
NEARFIELD BROADBAND ADAPTIVE BEAMFORMING
}

\author{
Thushara D. Abhayapala Rodney A. Kennedy \\ Telecommunications Eng. Group, RSISE \\ The Institute of Advanced Studies \\ Australian National University \\ ACT 0200 Australia
}

\author{
Robert C. Williamson \\ Dept. of Engineering \\ Australian \\ National University \\ ACT 0200 Australia
}

\author{
Darren B. Ward \\ School of Elec. Eng. \\ University College \\ UNSW, ADFA \\ ACT 2600 Australia
}

\begin{abstract}
A nearfield broadband adaptive beamforming approach based on the modal expansion of the solution to the spheri$\mathrm{cal}$ wave equation is formulated. It provides an efficient parameterization for the nearfield beamforming problem with a single parameter to focus the beamformer to a desired operating radius and a set of modal coefficients to control the angular response. As a consequence, the adaptive beamformer has a frequency invariant response for nearfield sources, decorrelates coherent interferers (thus combating signal cancellation) and has a smaller number of parameters to be adapted compared to a conventional beamformer. A simulation example is presented to demonstrate the use of the proposed method in microphone array applications in speech acquisition systems.
\end{abstract}

\section{INTRODUCTION}

Recently, there has been a growing interest in using microphone arrays in speech acquisition systems such as teleconferencing, hands-free mobile telephones in cars and hearing aids. For these applications the source of interest is located in the nearfield of the array, and the bandwidth required spans several octaves. Thus the array needs to not only operate over a wide band of frequencies, but also needs to cope with nearfield sources. It should also be capable of rejecting signals from undesirable sound sources, such as other talkers and reverberation noise. In this paper, we describe a nearfield broadband adaptive beamformer which addresses the above requirements.

Conventional adaptive beamformers such as the Frost beamformer [1] are found to be effective in the presence of strong interferers as long as the interferers are uncorrelated with the desired source and the assumption of plane wave propagation is met. In some cases, due to coherent interference, the conventional beamformers fail as a result of cancellation of the desired signal. This problem arises in speech acquisition using a microphone array, where the correlated interference is due to the reflections of the desired signal
[2]. Remedies have been proposed to reduce the effect of signal cancellation due to correlated interference by spectral averaging [2, 3]. However these works assume farfield sources.

Most of the work on adaptive microphone array systems in the literature either assumes plane wave propagation [4] or fails to address the issue of coherent interference [5]. In this paper, a nearfield adaptive beamformer is proposed to combat the signal cancellation due to correlated interference by extending and exploiting our earlier results [6].

\section{NEARFIELD BEAMFORMING}

Several methods of nearfield beamforming have been proposed recently $[6,7,8]$. Here we outline a method that permits an efficient parameterization which is well suited for nearfield broadband adaptive beamforming [6]

\subsection{Nearfield Modal Analysis}

Consider a theoretical continuous linear sensor aligned to the $x$ axis with an aperture illumination function $\rho_{r_{1}}(x, f)$, where $x$ is the distance to a point in the sensor from the sensor origin and $f$ is the frequency of operation. Also assume that this sensor can be focused to a radial distance $r_{1}$ from the sensor origin to give a desired frequency invariant angular response over the design bandwidth to sources at that distance. Thus the sensor spatial response to a source at an angle $\theta$ and distance $r$ from the array origin is given by

$$
b_{r_{1}}(r, \theta ; f)=\int \rho_{r_{1}}(x, f) \frac{1}{d(r, x, \theta)} e^{-i \frac{2 \pi}{c} f d(r, x, \theta)} d x
$$

where $i=\sqrt{-1}, c$ is the propagation speed of sound and $d(r, x, \theta)=\sqrt{x^{2}+r^{2}-2 r x \cos \theta}$ is the distance from the source to a point $x$ in the sensor. The beampattern (1) can be expressed using modes of the solution to the wave equation [6],

$$
b_{r_{1}}(r, \theta ; f)=\sum_{n=0}^{N} \beta_{n} \frac{R_{n}(r, f)}{R_{n}\left(r_{1}, f\right)} P_{n}(\cos \theta)
$$


when the aperture function is explicitly given by

$$
\rho_{r_{1}}(x, f)=\sum_{n=0}^{N} \beta_{n} \frac{1}{R_{n}\left(r_{1}, f\right)} j_{n}\left(\frac{2 \pi}{c} f x\right)
$$

where $\beta_{n}$ are the modal coefficients, $R_{n}(r, f) \triangleq$ $i \pi h_{n}^{(2)}\left(\frac{2 \pi}{c} f r\right), P_{n}(\cdot)$ are the Legendre functions, and $j_{n}(\cdot)$ and $h_{n}^{(2)}(\cdot)$ are the so called Spherical Bessel and Hankel functions of second kind respectively. Note that when $r=r_{1}$, beampattern (2) is frequency invariant and given by

$$
b_{r_{1}}\left(r_{1}, \theta, f\right)=\sum_{n=0}^{N} \beta_{n} P_{n}(\cos \theta), \forall \theta, \forall r_{1}, \forall f .
$$

Theoretically, to have any arbitrary frequency invariant beampattern over all frequencies, an infinite number of modes $N$ and an infinitely long sensor are needed. However it was shown in [6] that a reasonable beampattern specification over a frequency band consisting of a few octaves, can be realized using a finite number of modes with a finite length sensor.

\subsection{Discrete Array Structure}

Consider the output frequency response of the continuous sensor $\rho_{r_{1}}(x, f)$ for an arbitrary signal having Fourier Transform $z(x, f)$, impinging on the array at position $x$ :

$$
\hat{y}(f)=\int \rho_{r_{1}}(x, f) z(x, f) d x .
$$

We use the Trapezoidal rule to approximate (5) by

$$
\hat{y}(f) \approx \sum_{q=-Q}^{Q} g_{q} \rho_{r_{1}}\left(x_{q}, f\right) z\left(x_{q}, f\right),
$$

where $\left\{x_{q}\right\}_{q=-Q}^{Q}$ is a set of non-uniform discrete sensor locations (guidelines to choose these locations are given in [6]) and $g_{q}$ is the spatial weighting term. By combining (3) and (6) we write the output of the discrete array beamformer

$$
y(f)=\sum_{n=0}^{N} \frac{\beta_{n}}{R_{n}\left(r_{1}, f\right)} \sum_{q=-Q}^{Q} j_{n}\left(\frac{2 \pi}{c} f x_{q}\right) g_{q} z\left(x_{q}, f\right) .
$$

The above equation can be written in matrix form:

$$
y(f)=\mathbf{b}^{H} \mathbf{C}_{r_{1}}(f) \mathbf{z}(f)
$$

where ${ }^{H}$ denotes hermitian transpose, $\mathrm{b}=\left[\beta_{0}, \cdots, \beta_{N}\right]^{H}$ is a vector of modal coefficients,

$$
\begin{aligned}
\mathbf{C}_{r_{1}}(f) & =\operatorname{diag}\left[1 / R_{0}\left(r_{1}, f\right), \cdots, 1 / R_{N}\left(r_{1}, f\right)\right] \times \\
& {\left[\begin{array}{ccc}
j_{0}\left(\frac{2 \pi}{c} f x_{-Q}\right) & \cdots & j_{0}\left(\frac{2 \pi}{c} f x_{Q}\right) \\
\vdots & & \vdots \\
j_{N}\left(\frac{2 \pi}{c} f x_{-Q}\right) & \cdots & j_{N}\left(\frac{2 \pi}{c} f x_{Q}\right)
\end{array}\right] } \\
& \times \operatorname{diag}\left[g_{-Q}, \cdots, g_{Q}\right]
\end{aligned}
$$

and $z(f)=\left[z\left(x_{-Q}, f\right), \cdots, z\left(x_{Q}, f\right)\right]^{T}$ represents the array data. The importance of this formulation is that the discrete array beamformer (8) can be focused to any radius $r_{1}$ to give a frequency invariant response at that radius and there is a single set of modal coefficients $b$ which defines the spatial response over the bandwidth of interest. Thus the number of adaptation parameters is equal to the number of modes $N+1$ used. This is much less than that of a conventional adaptive beamformer where the number of adaptation parameters are equal to the product of the number of sensors and number of filter taps per each sensor.

\section{NEARFIELD BROADBAND MINIMUM VARIANCE BEAMFORMING}

The objective of broadband adaptive beamforming is to preserve a chosen frequency response for a desired signal arriving from a given source while minimizing the contribution from interfering sources. This is often achieved by minimizing the beamformer output power while maintaining the chosen frequency response in the look direction.

\subsection{Data Model}

Consider $V$ broadband signals impinging on a linear double-sided array of $2 Q+1$ sensors from nearfield sources at $\left(r_{1}, \theta_{1}\right), \cdots,\left(r_{V}, \theta_{V}\right)$ where $r_{v}$ is the distance to the $v$ th source from the array origin and $\theta_{v}$ is measured relative to the array axis. One of these signals is from the desired source at known location $\left(r_{1}, \theta_{1}\right)$, and the remaining signals are treated as interference. In this paper, we assume the desired source location is known exactly.

The received signals are discretized at each sensor and the resulting array data is divided into blocks of $J$ samples, and Discrete Fourier transformed to produce $M \leq J$ narrowband frequency bins within the design bandwidth $f \in\left[f_{L}, f_{U}\right]$, where $f_{L}$ and $f_{U}$ are the lower and upper frequencies of the design bandwidth respectively. The array data in the $m$ th frequency bin is

$$
\mathbf{z}\left(f_{m}\right)=\sum_{v=1}^{V} \mathbf{a}_{r_{v}}\left(\theta_{v} ; f_{m}\right) s_{v}\left(f_{m}\right)+\mathbf{n}\left(f_{m}\right),
$$

where $s_{v}(\cdot)$ is the signal emitted from the $v$ th source, $\mathbf{n}(\cdot)$ is the uncorrelated noise data and

$$
a_{r}(\theta ; f)=\left[\frac{e^{-i \frac{2 \pi}{c} f d(r, x-Q, \theta)}}{d\left(r, x_{-Q}, \theta\right)}, \cdots, \frac{e^{-i \frac{2 \pi}{c} f d\left(r, x_{Q}, \theta\right)}}{d\left(r, x_{Q}, \theta\right)}\right]^{T} .
$$

\subsection{Minimum Variance Beamforming}

We now apply the beamforming structure based on modal expansion (8) to the received data (10), giving the beam- 
former output

$$
y\left(f_{m}\right)=\mathbf{b}^{H} \mathbf{C}_{r_{1}}\left(f_{m}\right) \mathbf{z}\left(f_{m}\right)
$$

in the $m$ th frequency bin. The expected averaged output power of the beamformer over all frequency bins is

$$
\begin{aligned}
E\left\{|y|^{2}\right\} & =\frac{1}{J} \sum_{m=1}^{J} E\left\{\left|y\left(f_{m}\right)\right|^{2}\right\} \\
& =\mathbf{b} \overline{\mathbf{R}} \mathbf{b}^{H}
\end{aligned}
$$

where

$$
\overline{\mathbf{R}}=\frac{1}{J} \sum_{m=1}^{J} \mathbf{C}_{r_{1}}\left(f_{m}\right) E\left\{\mathbf{z}\left(f_{m}\right) \mathbf{z}^{H}\left(f_{m}\right)\right\} \mathbf{C}_{r_{1}}\left(f_{m}\right)^{H}
$$

is the frequency averaged data covariance matrix. The objective here is to find the modal coefficient vector $b$ that minimizes the interference power while passing signals from the desired location $\left(r_{1}, \theta_{1}\right)$ with unity gain for all frequencies within the design band. This is formulated as

$$
\begin{gathered}
\min _{\mathbf{b}} \mathbf{b}^{H} \overline{\mathbf{R}} \mathbf{b} \\
\text { subject to } \mathbf{D}^{H} \mathbf{b}=1_{M_{1}},
\end{gathered}
$$

where $\mathbf{D}=\left[\mathbf{C}_{r_{1}}\left(f_{1}\right) \mathbf{a}_{r_{1}}\left(\theta_{1} ; f_{1}\right), \cdots, \mathbf{C}_{r_{1}}\left(f_{M_{1}}\right) \mathbf{a}_{r_{1}}\left(\theta_{1}\right.\right.$ $\left.f_{M_{1}}\right)$ ] is the constraint matrix. Theoretically, a single constraint at the midband frequency should be sufficient. However the array bearnformer is only an approximation of the frequency invariant theoretical continuous sensor, thus a small number $M_{1}$ of constraints are needed across the frequency band of interest to guarantee a frequency invariant response [2].

Equation (16) is a well known constrained LMS problem and its optimum solution is given by [1]

$$
\mathbf{b}_{\text {opt }}=\overline{\mathbf{R}}^{-1} \mathbf{D}\left[\mathbf{D}^{H} \overline{\mathbf{R}}^{-1} \mathbf{D}\right]^{-1} \mathbf{1}_{M_{1}}
$$

Using (1) and (2) we can write the output of the continuous sensor (5) for the same signal environment described in section 3.1 as

$$
\hat{y}(f)=\sum_{v=1}^{V} \sum_{n=0}^{N} \beta_{n} \frac{R_{n}\left(r_{v}, f\right)}{R_{n}\left(r_{1}, f\right)} P_{n}\left(\cos \theta_{v}\right) s_{v}(f)+y_{n}(f)
$$

where $y_{n}(f)$ is the output due to sensor noise. Since the discrete array beamformer (12) is an approximation of the continuous sensor, it can be approximated using (18) as

$$
y\left(f_{m}\right) \approx \mathbf{b}^{H} \mathbf{T} \mathbf{U}\left(f_{m}\right) \mathbf{s}\left(f_{m}\right)+\mathbf{b}^{H} \mathbf{C}_{r_{1}}\left(f_{m}\right) \mathbf{n}\left(f_{m}\right)
$$

where the block diagonal matrix

$$
\mathbf{U}(f)=\operatorname{diag}\left[\left[\begin{array}{c}
\frac{R_{0}\left(r_{1}, f\right)}{R_{0}\left(r_{1}, f\right)} \\
\vdots \\
\frac{R_{N}\left(r_{1}, f\right)}{R_{N}\left(r_{1}, f\right)}
\end{array}\right], \cdots,\left[\begin{array}{c}
\frac{R_{0}\left(r_{D}, f\right)}{R_{0}\left(r_{1}, f\right)} \\
\vdots \\
\frac{R_{N}\left(r_{D}, f\right)}{R_{N}\left(r_{1}, f\right)}
\end{array}\right]\right]
$$

$\mathbf{T}=\left[\operatorname{diag}\left[P_{0}\left(\cos \theta_{1}\right), \cdots, P_{N}\left(\cos \theta_{1}\right)\right], \cdots, \operatorname{diag}\left[P_{0}(\cos \right.\right.$ $\left.\left.\left.\theta_{V}\right), \cdots, P_{N}\left(\cos \theta_{V}\right)\right]\right]$ and $\mathbf{s}(f)=\left[s_{1}(f), \cdots, s_{V}(f)\right]^{T}$.

To show the effects of frequency averaging in (15), we form an alternative expression for frequency average correlation matrix using (19) as

$$
\overline{\mathbf{R}}=\mathbf{T} \overline{\mathbf{R}}_{s} \mathbf{T}^{H}+\overline{\mathbf{R}}_{n},
$$

where $\overline{\mathbf{R}}_{s}=\frac{1}{J} \sum_{m=1}^{j} \mathbf{U}\left(f_{m}\right) \mathbf{R}_{s}\left(f_{m}\right) \mathbf{U}^{H}\left(f_{m}\right), \overline{\mathbf{R}}_{n}=$ $\frac{1}{J} \sum_{m=1}^{j} \mathbf{C}_{r_{1}}\left(f_{m}\right) E\left\{\mathbf{n}\left(f_{m}\right) \mathbf{n}^{H}\left(f_{m}\right)\right\} \mathbf{C}_{r_{1}}^{H}\left(f_{m}\right)$ and $\mathbf{R}_{s}=$ $E\left\{\mathbf{s}\left(f_{m}\right) \mathbf{s}^{H}\left(f_{m}\right)\right\}$. Observe that the frequency averaging preserves the directional information of all sources which are stored in matrix $\mathbf{T}$ and uses radial information in $\mathbf{U}\left(f_{m}\right)$ to reduce any signal correlation present in $\mathbf{R}_{s}\left(f_{m}\right)$ by forming the weighted frequency average source correlation matrix $\overline{\mathbf{R}}_{\boldsymbol{s}}$.

\section{ADAPTIVE NEARFIELD BEAMFORMING}

The constrained minimization problem (16) is in the same form as that considered by Frost [1] where he optimized the array weights whereas we have optimized the modal coefficients. In our case, we use Frost's gradient-based algorithm, which converges in the mean to the optimum solution (17), to adapt the modal coefficients.

In summary, the algorithm for our case is given by

$$
\mathbf{b}(k+1)=\mathbf{P}[\mathbf{b}(k)-\mu \hat{\overline{\mathbf{R}}} \mathbf{b}(k)]+\mathbf{F}
$$

where $\mathrm{b}(k)$ is the set of modal coefficients at the $k$ th iteration, $\mathbf{F}=\mathbf{D}\left(\mathbf{D}^{H} \mathbf{D}\right)^{-1} 1_{M_{1}}$, the $(N+1) \times(N+1)$ matrix $\mathbf{P}=\mathbf{I}-\mathbf{D}\left(\mathbf{D}^{H} \mathbf{D}\right)^{-1} \mathbf{D}^{H}$, the initial value of modal coefficients $\mathbf{b}(0)=\mathbf{F}$,

$$
\hat{\overline{\mathbf{R}}}=\frac{1}{J} \sum_{m=1}^{J} \mathbf{C}\left(r, f_{m}\right) \mathbf{z}\left(f_{m}\right) \mathbf{z}^{H}\left(f_{m}\right) \mathbf{C}^{H}\left(r, f_{m}\right)
$$

is an $(N+1) \times(N+1)$ matrix used to estimate $\overline{\mathbf{R}}$ for the $k$ th iteration, and $\mu$ is the adaptive step size parameter which controls the convergence characteristics of the algorithm. As noted by Frost, $\mu$ is chosen such that $0<\mu<1 / \sigma_{\max }$, where $\sigma_{\text {max }}$ is the largest eigenvalue of $\mathbf{P} \overline{\mathbf{R}} \mathbf{P}$.

\section{EXAMPLES}

We now present an example to show the performance of the adaptive array in a signal environment which consists of nearfield sound sources and reverberation noise.

The design is suitable for speech with a design bandwidth of $600-3000 \mathrm{~Hz}$ and the maximum mode index $N=11$ is used. According to [6], we determine the number of sensors needed and their location to meet the above bandwidth and mode specification. The resulting nonlinear double sided array has total of 39 sensors with aperture length of $2.6 \mathrm{~m}$. 


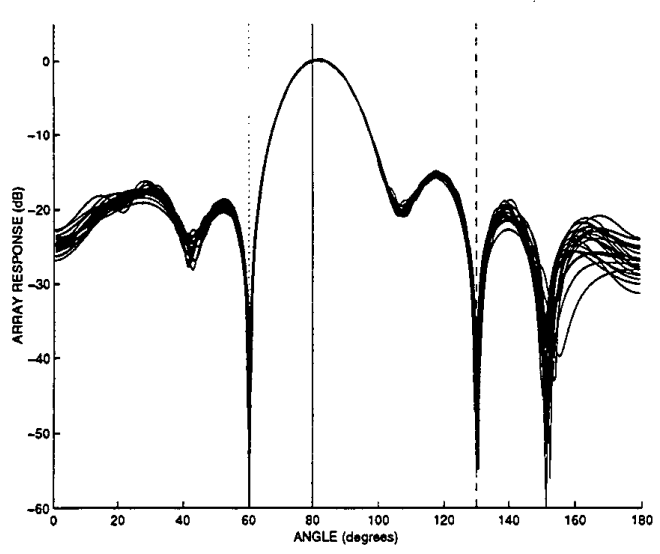

Figure 1: Spatial response of the adapted beamformer at $5 \mathrm{~m}$ from the array origin at 26 frequencies within the design band of $500-3000 \mathrm{~Hz}$.

The signal environment consisted of a desired source and two uncorrelated interference sources, all $5 \mathrm{~m}$ away from the array origin, with flat frequency spectra over the bandwidth of interest. The desired signal was at $80^{\circ}$, the first interferer was at $130^{\circ}$ and 10 times stronger than the desired signal, and the other interferer was at $60^{\circ}$ with the same power as the desired source. White Gaussian noise, uncorrelated from sensor to sensor, was modelled at the input of each sensor such that the SNR of the desired signal at the origin is $0 \mathrm{~dB}$. The received array data $\mathbf{z}\left(f_{m}\right)$ was constructed for 26 frequency bins uniformly spaced within the desired bandwidth, and the adaptive algorithm was used to find the modal coefficients with $M_{1}=3$ frequency constraints. The adaptation step size $\mu=1 \times 10^{-3}$ was used. Figure 1 shows the beampatterns at $r=5 \mathrm{~m}$ produced by the adapted modal coefficients after 4000 data samples. Observe that the beampattern has nulls in the directions of interferers and exhibits little variation with frequency at the focussed radius.

Next we consider the performance of the adaptive beamformer in a reverberant rectangular room $(7 \mathrm{~m} \times 10 \mathrm{~m})$ with reflection coefficient of 0.8 (the array is aligned to the $7 \mathrm{~m}$ long wall). We used the image-source method to calculate the positions of images (up to the 10th order) of 3 sound sources due to 4 vertical walls. The adapted beampatterns after 4000 data samples are given in Figure 2. Note that, even with the highly correlated reflected signals, the beamformer is able maintain a unity gain in the desired direction and attenuates the other two interference sources while preserving its frequency invariant behaviour at the focused radius in the nearfield. Unsurprisingly, the performance is not quite as good as in Figure 1.

Acknowledgement: This work was supported by the Australian Research Council.

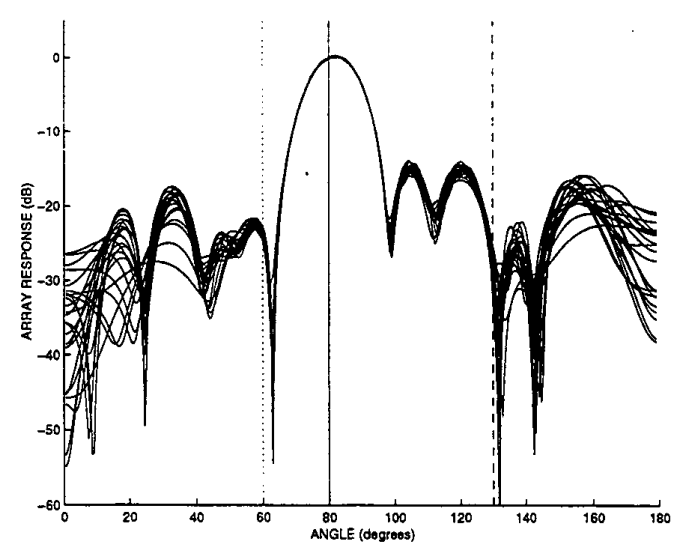

Figure 2: Spatial response of the adapted beamformer at $5 \mathrm{~m}$ from the array origin at 26 frequencies within the design band with the effects of reverberation.

\section{REFERENCES}

[1] O.L. Frost III, "An algorithm for linearly constrained adaptive array processing," Proc. IEEE, vol. 60, pp. 926-936, Aug. 1972.

[2] D.B. Ward, "Technique for broadband correlated interference rejection in microphone arrays," IEEE Trans. Speech and Audio Proc., vol. 6, pp. 414-417, July 1998.

[3] A. Zeira and B. Friedlander, "Interpolated array minimum variance beamforming for correlated interference rejection," Proc. IEEE Int. Conf. Acoust., Speech Signal Processing, pp. 3165-3168, May 1996.

[4] K. Farrell, R. J. Mammone , and J. L. Flanagan, "Beamforming microphone arrays for speech enhancement," Proc. IEEE Int. Conf. Acoust., Speech Signal Processing, pp. 285-288, May 1992.

[5] Y. Grenier, "A microphone array for car environments," Speech Communication, vol. 12, pp. 25-39, Mar. 1993.

[6] T.D. Abhayapala, R.A. Kennedy, and R.C. Williamson, "Nearfield broadband array design using a radially invariant modal expansion," J. Acoust. Soc. Amer, accepted Aug 1998.

[7] R.A. Kennedy, T.D. Abhayapala, and D.B. Ward, "Broadband nearfield beamforming using a radial beampattern transformation," IEEE Trans. Sig. Proc., vol. 46, pp. 2147-2156, Aug. 1998.

[8] J. G. Ryan and R. A. Goubran, "Nearfield beamforming for microphone arrays," Proc. IEEE Int. Conf. Acoust., Speech Signal Processing, pp. 363-366, May 1997. 